\title{
ProMED Android Mobile Application for finding the Availability of Medicine
}

\author{
Praveena B, Abhishek, Sowmya Nag K \\ Electronics and Communication Engineering \\ R V College of Engineering \\ Bangalore, India
}

\begin{abstract}
This paper proposes on how the development of an android application helps the current society on accessing various medicine and drugs within a few moments before they reach the medical shops and markets. Developments in engineering tools and technology are resolved many problems in the current world. All the issues and challenges are being tackled in computer science and engineering concepts with algorithms that lead to the development of web, android, and iOS applications. The availability of medicines and the reachability to the people who are in need and emergency cases were not an easy process which leads to coming up with this project idea and concept. This android and web app have used Google maps API, other Android SDK, and angular materials for the development. This app takes the data from user surrounding area shops for availability and suggests users the same via the application. It will discuss how this can overcome the scenario with the help of APIs.
\end{abstract}

Keywords-Android, MongoDB, Android Architecture, native development, material design

\section{INTRODUCTION}

Android is an opensource framework and mobile operating system. IoT can create a very smart environment using smart devices. Paired with mobile applications that enable users to control these devices remotely, this technology is one of the most remarkable technologies of this era. IoT-based mobile applications have a wide range of benefits. A few of these benefits are Cost-effective, Knowledge of consumer behavior, Enhanced productivity, Improved customer experience, safer workplaces, Data assortment and analysis, and Research.

The ways through which mobile apps are influencing the IoT sector and making the technology more diverse and versatile. Some of the major examples are Smart Homes, Wearables, Healthcare and Medicine, Retail, Agriculture, and Smart Cities. This paper introduces the app architecture and working of the application with all its functionalities which is developed using an android native approach with MVVM architecture.

\section{PROMED APP}

This Android application helps the users in finding the availability of the medicines in nearby stores. Before we know about how the application looks, we will know how the application is designed and built to meet the requirements. The requirements are to get the location of the user device by taking the help of the Google Maps APIs and the iframe data that helps in building the web application for the database. Another requirement is to store the user details and all the medical stores available stock of all the medicine and their details. The app should display all the details about the medicine that is the user looking for in the application and its direction of use. The application should allow the users to take any emergency help from the healthcare centers especially aimed for senior citizens and handicapped people.

The application works presently with the android smart devices and with the functionalities that are met. All the users and medical store data are being stored in the MongoDB server for the demo project of the android application and it is discussed in the next section of the paper.

\section{MONGODB SERVER}

For the web application on the server part, we are using this opensource framework to operate with the data. The bean java class in the project defines the fields of MongoDB collection and they are case sensitive. Then the field names and inputs will be taken from HTML and TS files of angular which are binding with the database.

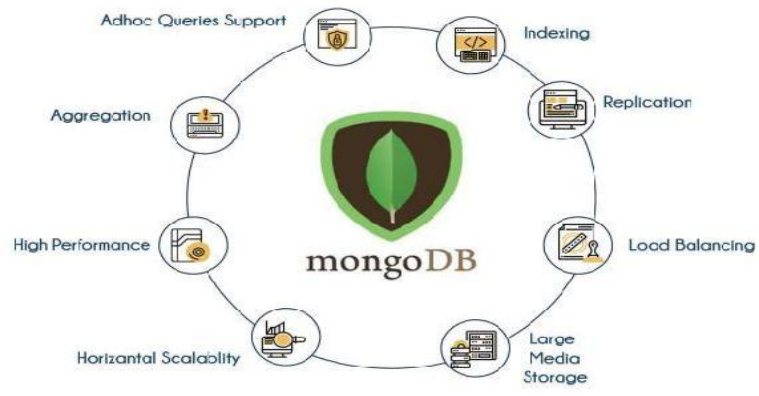

Fig -1: MongoDB Features

Java bean class name and collection name of the MongoDB should be the same or else data will not be in binding with the database. Collection data of our project can be updated from the UI of our web application or via

JSON file directly importingin the server and can be updated from the REST API platforms like POSTMAN and Insomnia in JSON body with post mapping.

\section{DESIGN AND FEATURES}

The features and the design of the app are shown below which explains the advantages of the app and the things that are being overcome from the project. 


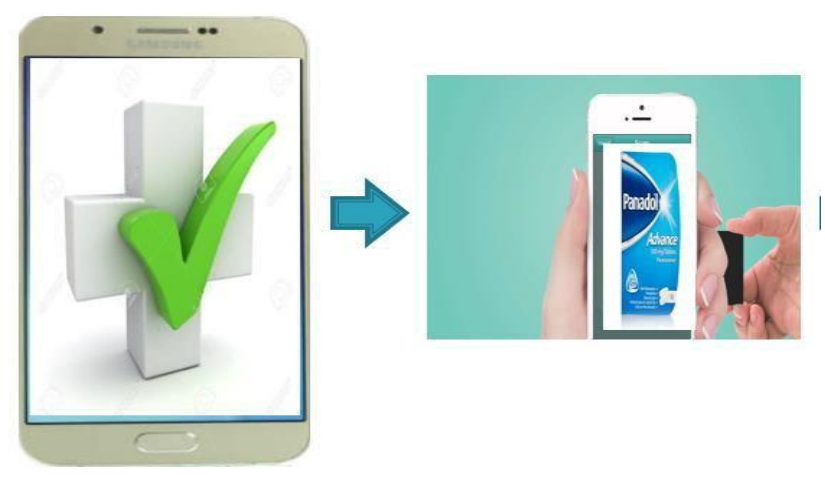

Fig -2: App Demo-1

Figure App Demo-1 shows how the app looks when the user opens it and starts to use it. In the same figure, we can notice that the scanning of the medicines which are going to be added as in the future to the application. When the user clicks on the app it asks for permission for accessing the location and then it gives an option for searching the medicine. After the user search in the app then it connects to the backend server project and by the help of location and the database it gives the results in the android page to the user with a google app on showing the location of the medical shop that the medicine stock is available in.

Later it gives the feedback to the administrator in the server. Then this server requests the shop owners to update the database with the stocks that are available with them and they do to increase their marketing and popularity in business.

For this project demo as it is initial stages of the application more than 20 medicals store data have been stored in the local MongoDB server to showcase the working of the application. Some Common Mistakes

And the survey has been made to collect their feedback on what are the features they like to see in our project and what would be the ways they can update their data and the importance of healthcare in society.
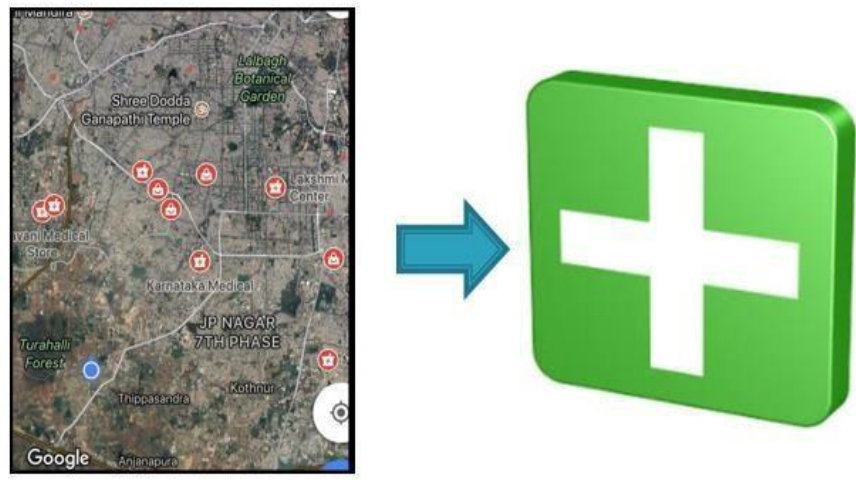

The user gets the google map page in this android application when he/she search the medicine in the app. When the application servers get the data from the database on availability it links with the Google APIs to display the available medical shops for the searched medicine.

This serves the purpose of the application in this initial phase of the project. The conclusion and the future scope and the gaining from the project are discussed in the next section.

\section{CONCLUSION}

From this project, we learned how to make the idea into action and then implement it with real-world problems. The main conclusion that we made was every problem of the world is an opportunity to overcome in your way and its on how we implement something that will be useful to a wider community of people.

In the future, it is planned to add image processing algorithms and techniques. Then in the application will have the features like scanning the medicine with the camera in the android application and give the results in addition to type and search option. Further features like handwritten recognition modules can be added to find the medicine and the date of prescriptions that the user got from doctors and knowing the medical stores where all of them available instantly. Another major feature that discussed was voice input recognition which covers all the community of the people if they are physically challenged and not able to type or scan the data.

Adding healthcare contacts and support will be a good feature to add to the project. This project can be taken as a reference to further research on the healthcare problems that arise shortly who are going to help the society by designing such helpful apps.

\section{REFERENCES}

[1] A. Kathuria and A. Gupta, "Challenges in Android Application Development: A Case Study" International Journal of Computer Science and Mobile Computing, vol. 4, no. 5, pp. 294-299, May 2015

[2] OL. Google Android Developers, Android Develop Guide, https://developer.android.com/guide/platform.

[3] Ribeiro and A. R. D. Silva, "Survey on Cross-Platforms and Languages for Mobile Apps," Eighth International Conference on the Quality of Information and Communications Technology, 2012.

[4] ] Jianye Liu; Jiankun Yu, Research on Development of Android Applications, International Conference on Intelligent Networks and Intelligent Systems,2011.

[5] Pohares, V. C. Kulloli, T. Bhattacharyya, and S. Bhure, "Cross Platform Mobile Application Development," International Journal of Computer Trends and Technology, vol. 4, no. 5, pp. 1095-1100, 2013.

[6] J. Dongjiu Geng, Yue Suo, Yu Chen, Jun Wen, Yongqing Lu, Remote Access and Control System Based on Android Mobil Phone'vol.2. Journal of Computer Applications, pp. 560-562, 2011.

Fig -3: App Demo-2 